\title{
RADIO COMMUNICATION AS APPLIED TO FOREST PROTECTION.*
}

\author{
By J. Stuart Richardson \\ Northern Electric Company
}

$\mathrm{T}^{\mathrm{H}}$

HE history of the growth of communication is a history of the world's progress. Communication has contributed a measure of safety to what ever specific purpose it has been applied. The early inhabitants of the earth used smoke signals to save themselves the effort of journeys, and to give warning to their kind of impending danger. A system of flag signals was developed for use by ships, so that they might not become endangered either by proximity to each other or to the shore. The social order of today is built up on elaborate and specialized systems of communication, the main object of each of which is saving, either of time, property, or life.

The purpose of this paper is to consider in what measure radio may contribute to the problem of communication for forest protection. At the present time various methods are in use in order that the maximum area may be under the observation of the minimum number of observers. Look-out towers, aeroplanes and ground patrols are used most frequently for this purpose, and incidentally it is possible that the knowledge of their presence in the bush tends to create a more careful attitude in hunters, prospectors and others who might be careless in the creating of fire hazards.

During the summer months every effort is made to warn the public of the danger of causing forest fires. Newspaper warnings, posters, and almost every conceivable means are used to bring home to those who, by thoughtlessness, might be the means of destroying large areas of timber, with consequent danger to life and property. Such warnings have undoubtedly produced excellent results, but although it is probably safe to say that the public are more keenly aware of the risks involved as a result of their activities in the bush, the danger of fire is still one which requires the gravest consideration. Apart from man made fires, lightning and spontaneous combustion are of course additional, re-causing hazards to which the bush is subject.

It is therefore essential, if the bush is to be protected against fire, that means must be adopted in order that fire may be detected and dealt with in its early stages. In brief, a system of communication which will meet special requirements, is necessary.

The difficulties of providing communication through forest country must be apparent to all who have any knowledge of the conditions. There are many methods, which, under special conditions, will provide a means of communication. Each method, however, possesses certain inherent weaknesses. Perhaps the oldest means by which information may be conveyed

* Paper read before the Woodland Section of the Canada Pulp and Paper Association. 
from one point to another is by messenger. The most modern and technically complex is the wireless telephone and telegraph. A study of the kind of messages, the men who send them, and the ranges over which the communication system must operate, leads inevitably to the conclusion that the telephone and radio telephone come nearer to meeting all requirements. The telegraph and radio telegraph have certain advantages over the corresponding telephone facilities in that greater distances can be spanned, but due to the fact that a knowledge of code is necessary, this advantage cannot generally be capitalized.

To some extent the ordinary wire telephone has already been adapted for bush operations. To be reliable, however, a "right of way" has to be estab. lished in order that falling trees may not damage the line. Such lines are costly to build and to maintain in a good state of repair. They are also subject to damage during a fire with consequent interruptions to the service. Admittedly, the "right of way" provides a great deal of protection from the effects of a fire, but even though this be wide, sufficient heat may be present to crack the insulators, and to cause the wire either to break or become grounded. If no "right of way" is provided then these conditions become aggravated to such an extent that the line could hardly be considered to be of much practical use except under ideal conditions.

The very nature of radio communication is such as to make it free from many of the weaknesses of wire lines, particularly when assigned to service in bush country. The communication medium by which radio is possible cannot be affected by conditions which would destroy a wire line and render its service useless. The medium of radio is everywhere available. No "right of way" has to be obtained and cleared. No lakes, mountains and other natural barriers have to be circumvented. With suitable types of equipment the points of communication can quite readily be moved to meet needs that may arise from time to time; a condition quite in contrast to that where telephone lines are used, as the investment is almost a total loss if for any reason its use is discontinued.

One point of outstanding significance can be credited to radio in that direct communication may be had with ships and aeroplanes either stationary or in motion. In certain areas use has been made of boats for forest protection operation. Furthermore, the rapid means of fire ranging and transportation of fre fighting equipment afforded by aircraft make them ideal for the protection of large areas of valuable wooded country, and particularly so when augmented by means of radio communication. One can readily appreciate what a saving in time can be made by a radio message from a plane say 40 or 50 miles from headquarters to the effect that a fire is raging in a certain area. Men and equipment can be assembled and immediately dispatched to 
the fire area and the fire ranging plane may continue its flight over other areas. Without any means of communication the plane would have to return to headquarters to report the fire, with consequent loss of time both in the quenching of the fire, and in the ranging of further territory.

Radio communication between aircraft and ground stations has been developed to a great extent during the past two or three years and the success with which it has been applied is widespread. Most of the large aircraft travel and transport companies have their planes radio equipped, and the majority of large airports are provided with equipment for communicating with planes flying over established routes. The equipment developed for this purpose is of course equally suitable for use in planes employed in fire ranging services. The ground station equipment used at airports would also be suitable for use at patrol headquarters in fire ranging areas. Furthermore, the transmitting equipment designed for use in aircraft has had to meet more rigid requirements than that for any other field of radio communication. It has had to be essentially dependable, simple, sensitive and light. Dependable, because no adjustment is possible in flight. It may be necessary to operate the equipment for a few seconds at a time only, or for the whole period of flight, yet it must function efficiently at all times if it is to fulfil its purpose. Secondly, it must be simple to control, so that its operation demands little concentration from the pilot, who is, in the majority of cases, also the operator. Thirdly, it must be highly sensitive to compensate for the limitations of aircraft antennae, which are relatively poor in comparison with those that may be constructed at ground stations. Fourthly it must be light, for every pound of navigating equipment in a commercial plane means a reduction in the amount of "pay load" which may be carried. Other important requirements of aircraft radio equipment are compactness and ruggedness. We submit that all the requirements encountered in the designing of radio equipment for aircraft will apply in almost equal measure to radio equipment for bush operations.

It would seem logical to presume that the future of forestry protection will see an increase in the use and co-operation of both aircraft and radio.

Radio communication commends itself as an adjunct to any means of fire ranging. In many places towers located at strategic points are used for observation purposes, and by this means information is gathered as to the location of fires. Obviously a radio system with equipment at or near these towers would increase to a large extent the effectiveness of this type of fire ranging. By exchanges of information between say three points as to the direction in which a given fire is located from each tower it would be possible in a few minutes to establish the exact location of the fire.

Communication has a number of different applications in connection with 
forestry protection. It may be used to advise rangers of the entry into the forest of campers, prospectors or others who may be the cause of fire hazard. It provides the means whereby the location of fire may be reported at the earliest passible moment. It enables headquarters to keep in touch with developments, so that the best use may be made of both men and materials. There are doubtless further ways in which the exchange and dispatching of information by radio could be used to great advantage in fire ranging. Once a communication system is set up for any purpose there always develops increased uses for such a system, and those who are familiar with and expert in the technique of forest protection can undoubtedly develop many ways of taking advantage of the flexibility of radio communication.

It must not be inferred from what has been said above that radio is entirely free from limitations in its use for forest protective work, and that equipment for all conditions is available. It must be recognized that while the transmission medium of radio is not subject to hazards which might destroy or impair wire telephone circuits, it is nevertheless subject to certain atmospheric conditions over which no control can be as yet exercised. Static interference and fading must be contended with in radio communication, but by the careful location of equipment, and by the obtaining of a suitable operating frequency, these disturbing conditions can be reduced to a minimum.

The question of power for energizing radio equipment is a matter of prime importance, especially under conditions where the portability is of great concern. There are now available, however, gasoline driven power plants of sizes suitable to almost any need, and these are of compact efficient and rug. ged design, thus lending themselves admirably to this particular work. In this connection it might be observed that the adoption of a suitable calling schedule would result in a conservation of power.

Within the past few years there have been developments in radio equipment which are of interest when considering the application of radio to forestry protection. Outstanding among these is the use of a quartz crystal for the control of frequency. By this means the operation of a transmitter is simplified, and transmission is maintained within $.025 \%$ of the allotted frequency. A receiver may be "set" for this particular frequency, and after the initial adjustments have been made, it will only afterwards be necessary to apply power thereto to hear the desired station. Furthermore, radio re: ceivers with automatic volume control are now available which ensure that the phenomena known as "fading," which has for a long time proved a handicap to radio reception, is reduced to minimum.

In spite of the advances that have been made toward the simplifying of radio apparatus, a higher degree of intelligence is required for its efficient operation than is required for the operation of a line telephone. That this 
consideration is not a serious drawback to the use of the radio can be seen from the increasing uses to which the radio telephone has been applied during the past few years. Radio has been successfully applied to the operation of aircraft, police, coastal and fireboats, and to a certain extent to forest patrol work.

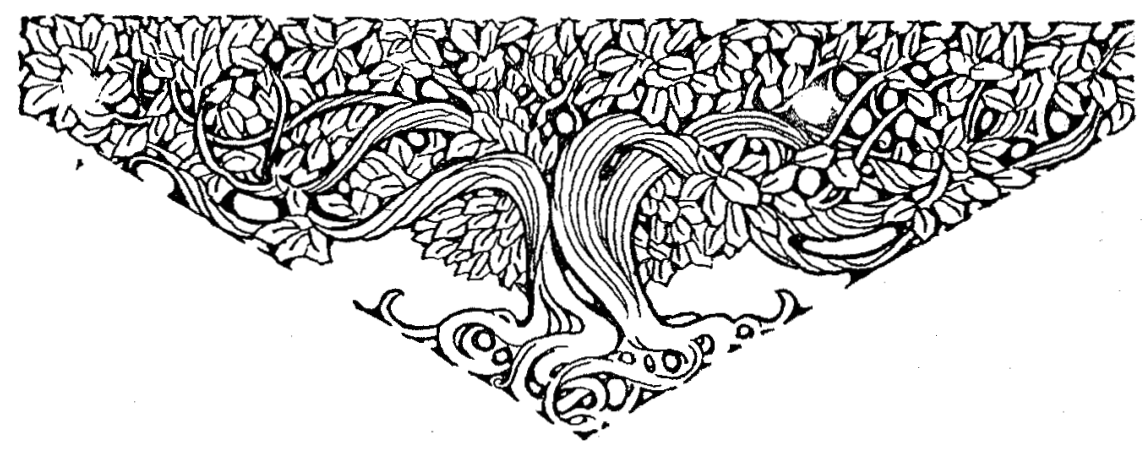

